\title{
C2 and the Kuramoto Model: An Epistemological Retrospective
}

\author{
$\underline{\text { A. H. Dekker }}^{\text {a }}$ \\ ${ }^{a}$ Dekker Consulting, P.O. Box 3925, Manuka, ACT, 2603, Australia \\ Email: dekker@acm.org
}

\begin{abstract}
The Kuramoto Model is a simple model of synchronisation phenomena within networks of coupled oscillators. The model has been applied to military Command and Control (C2), as illustrated in Figure 1. The mapping between model and reality is summarised in Table 1.

In this paper we briefly summarise the Kuramoto Model, and report a simple agent-based combat simulation experiment justifying the applicability of the model to $\mathrm{C} 2$. In the experiment, a networked blue force consisting of 8 infantry fighting vehicles (supported by artillery) attempts to move through a region containing a red force of 40 units. There is a statistically significant monotonic nonlinear relationship between angle correlations in the Kuramoto Model and plan-agreement correlations in the combat simulation.

We conclude the paper with an epistemological reflection on the use of the Kuramoto Model, and the lessons that can be drawn from it for computer simulation of human processes, especially in the military domain.
\end{abstract}
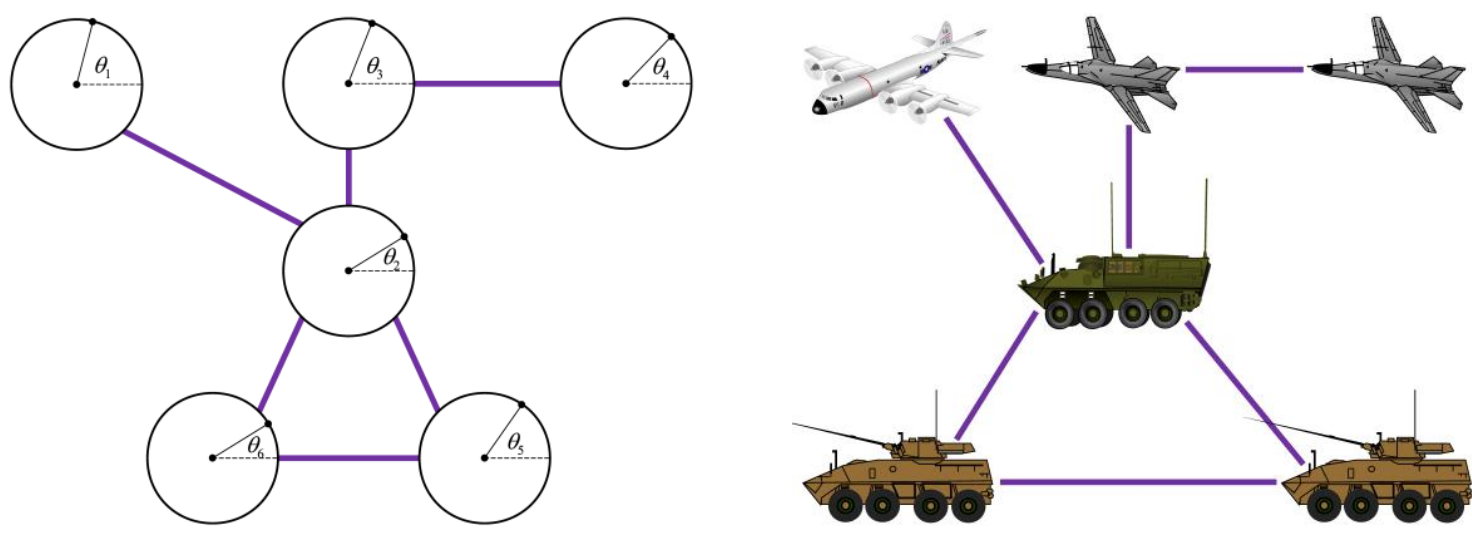

Figure 1. Model and reality: a network of oscillators (left) models a network of military nodes (right).

Table 1. Mapping the Kuramoto Model to the $\mathrm{C} 2$ domain.

Kuramoto Model

C2 application

Network of nodes with angle $\boldsymbol{\theta}$ in each node

Difference between angles in different nodes

Feedback given by differential equation acts to reduce differences between angles

Short network paths (low average distance) and multiple alternate network paths (high connectivity) support synchronisation

Problems in synchronising large cycles (artefact)

\section{$\longleftrightarrow \quad$ Network of nodes with plan in each node \\ Level of conflict between plans in different nodes}

Feedback given by $\mathbf{C 2}$ processes acts to reduce level of conflict between plans

Short network paths (low average distance) and multiple alternate network paths (high connectivity) support synchronisation

Many C2-relevant properties of synchronisation with no counterpart in the Kuramoto Model

Keywords: $\quad$ Kuramoto Model, NetLogo, networks, synchronisation, command and control, epistemology 


\section{INTRODUCTION}

The Kuramoto Model (Kuramoto 1984; Strogatz 2000; Dorogovtsev et al. 2008) is a simple model of synchronisation phenomena. The model was originally formulated in the physical sciences, where a network of oscillating entities can lock on to a common frequency. It has also been applied to biological synchronisation (Winfree 1980) and to synchronisation in military organisations (Dekker 2007, 2010; Kalloniatis 2008, 2010; Kalloniatis \& Zuparic 2014, 2016).

A typical network of coupled oscillators is shown in Figure 1(a). Each node has an associated phase angle $\theta_{i}$, as well as its own natural frequency $\omega_{i}$ (assumed to come from a unimodal and symmetric distribution). The model dynamics are governed by the following differential equation, which describes a negative feedback process: differences between $\theta_{i}$ and $\theta_{j}$ act to bring $\theta_{i}$ and $\theta_{j}$ closer together:

$$
\dot{\theta}_{i}=\omega_{i}+k \sum_{j=1}^{n} A_{i j} \sin \left(\theta_{j}-\theta_{i}\right), \quad i=1, \ldots, n .
$$

Here $A_{i j}$ is the adjacency matrix of the network topology and $k$ is a coupling constant which determines the strength of the coupling. In this paper we will consider the network topologies in Figure 2, which differ in the average distance (mean path length) $D$ between nodes, and the vertex connectivity $K$ (minimum number of independent paths between nodes). The degree of synchronisation within the network can be measured by the correlation coefficient $r$ :

$$
r=\sqrt{\left(\frac{\sum_{i} \sin \theta_{i}}{n}\right)^{2}+\left(\frac{\sum_{i} \cos \theta_{i}}{n}\right)^{2}}
$$

As a theory of synchronisation in Command and Control (C2), the Kuramoto Model is applied as in Figure 1 and Table 1 (Dekker 2013a). The network of oscillators corresponds to a network of military nodes with the same topology. The angles $\theta_{i}$ themselves do not correspond to any quantity in the military world, but the differences $\theta_{i}-\theta_{j}$ correspond to the level of conflict between plans in different military nodes.

The negative feedback process in Equation 1 corresponds to the real-world $\mathrm{C} 2$ processes which act to reduce level of conflict between plans in adjacent nodes, i.e. to ensure that plans are synchronised.

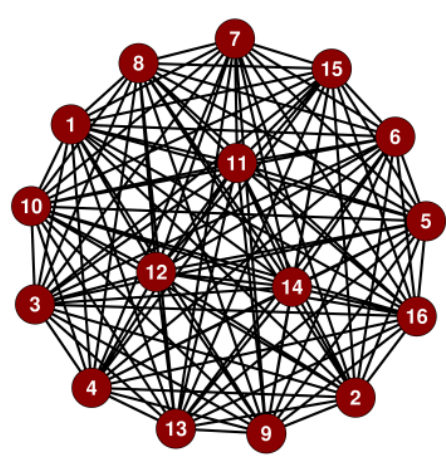

(a) Complete $(D=1, K=15)$

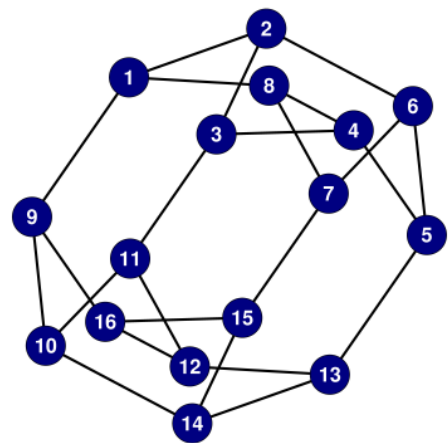

(d) Cubic $(D=2.2667, K=3)$

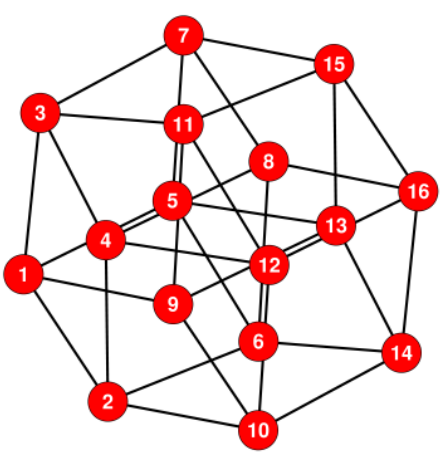

(b) Hypercube $(D=2.1333, K=4)$

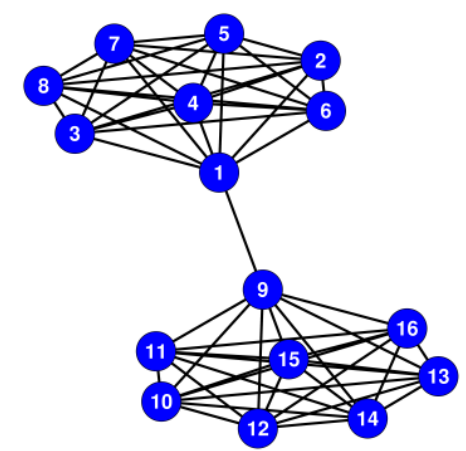

(e) Dumbbell $(D=1.9333, K=1)$

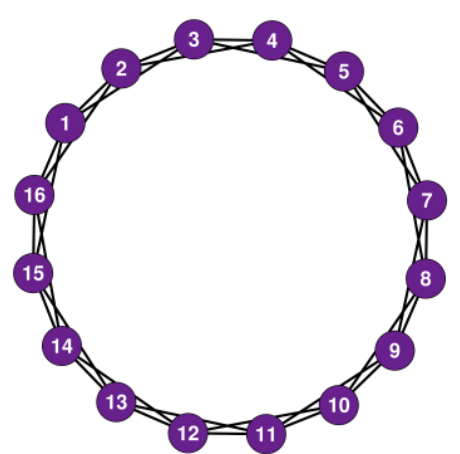

(c) Antiprism $(D=2.4, K=4)$

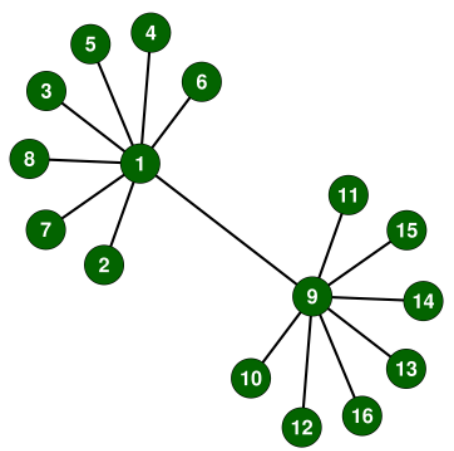

(f) $\operatorname{Tree}(D=2.2833, K=1)$

Figure 2. Six 16-node networks, with their average distance $(D)$ and connectivity $(K)$. The dumbbell and tree topologies provide a simple model of traditional $\mathrm{C} 2$ structures. 
The correspondences in Table 1 are not merely metaphors. Figure 3 (redrawn from Dekker 2013a) shows that there is a monotonic correspondence between candidate solutions to the Assignment Problem and sets of Kuramoto Model angles, such that better solutions correspond to higher correlation coefficients $r$. As an additional piece of evidence, in this paper we will report an experiment showing that there is also a correspondence between Kuramoto Model dynamics and a simple agent-based model of a military task.

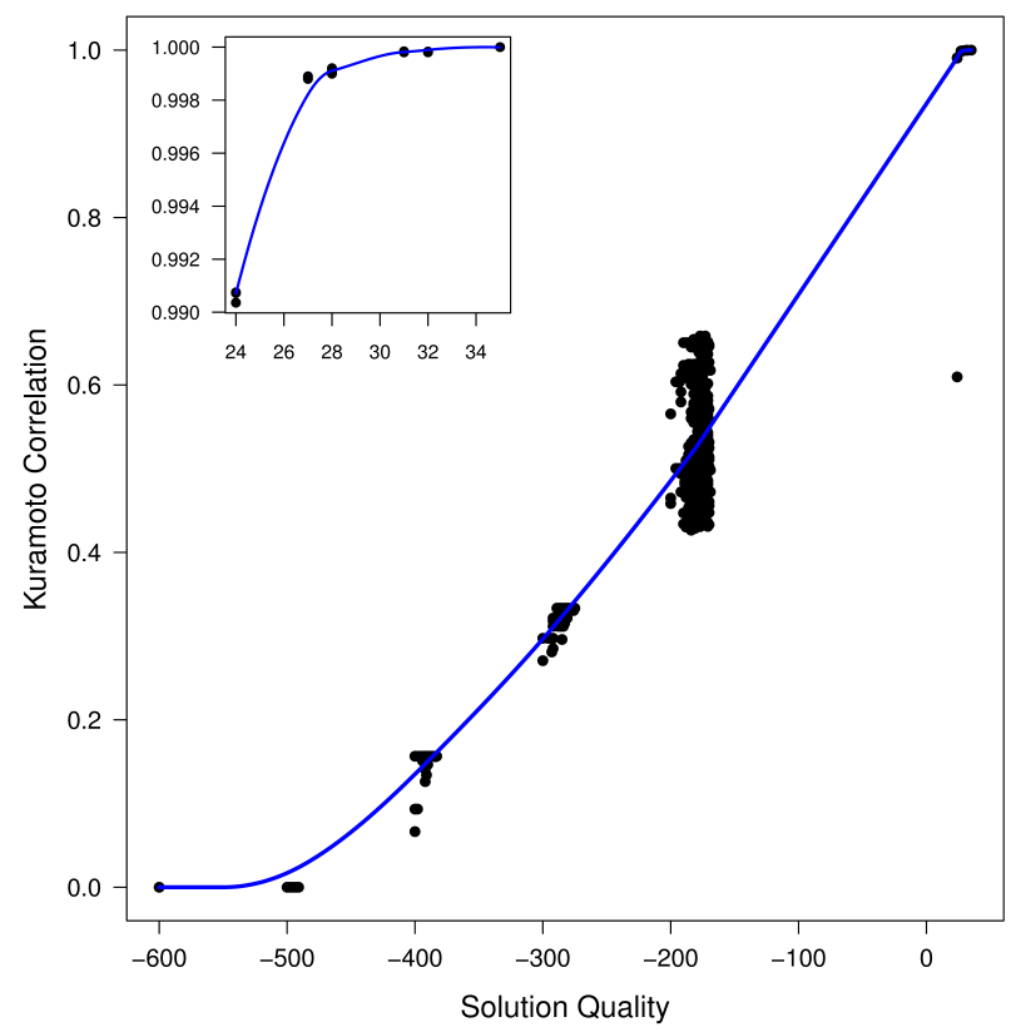

Figure 3. Assignment Problem solutions correspond to Kuramoto angle sets (redrawn from Dekker 2013a).

Of course, there are many properties of military synchronisation with no counterpart in the Kuramoto Model. There is also a model artefact: the dynamics in Equation 1 result in difficulties synchronising large cycles (see Figure 4). This difficulty results from an interaction between the angles $\theta_{i}$ and positions of nodes within a large cycle. This difficulty does not correspond to any real-world military phenomenon, because the angles $\theta_{i}$ do not correspond to any quantity in the military world.

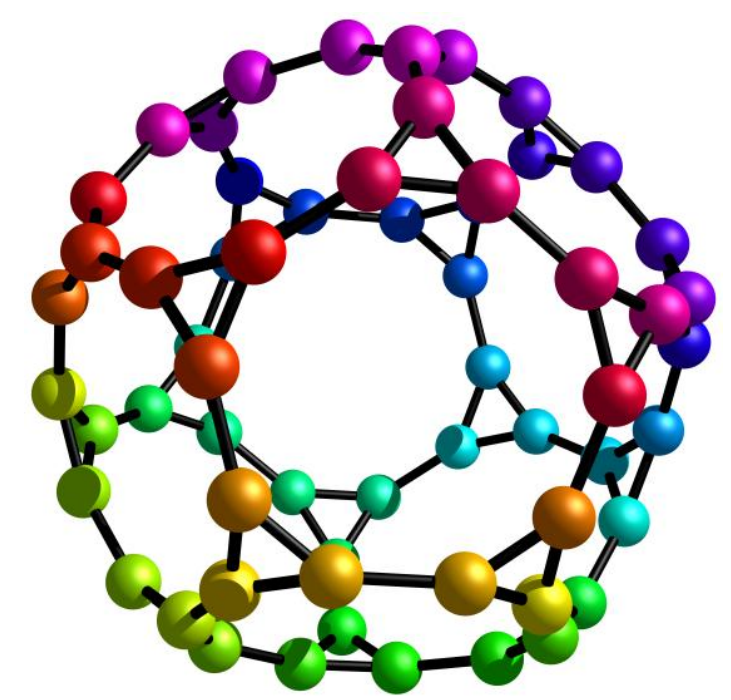

Figure 4. Unsynchronised stable state for a Kuramoto network containing large cycles. Here the angles $\theta_{i}$ are visualised as colours on a red-orange-yellow-green-blue-purple-red colour wheel. 
Dekker, C2 and the Kuramoto Model: An Epistemological Retrospective

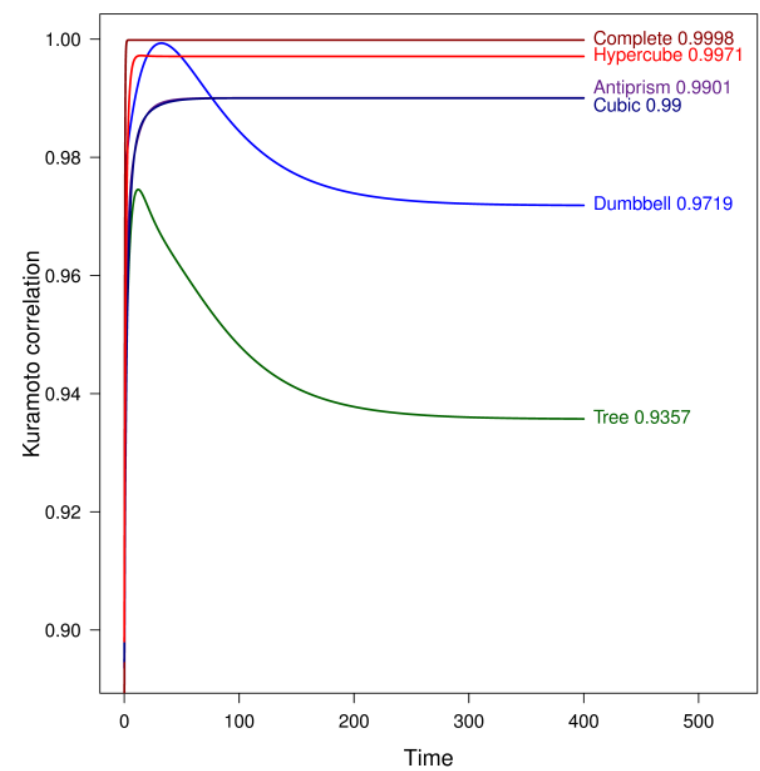

(a)

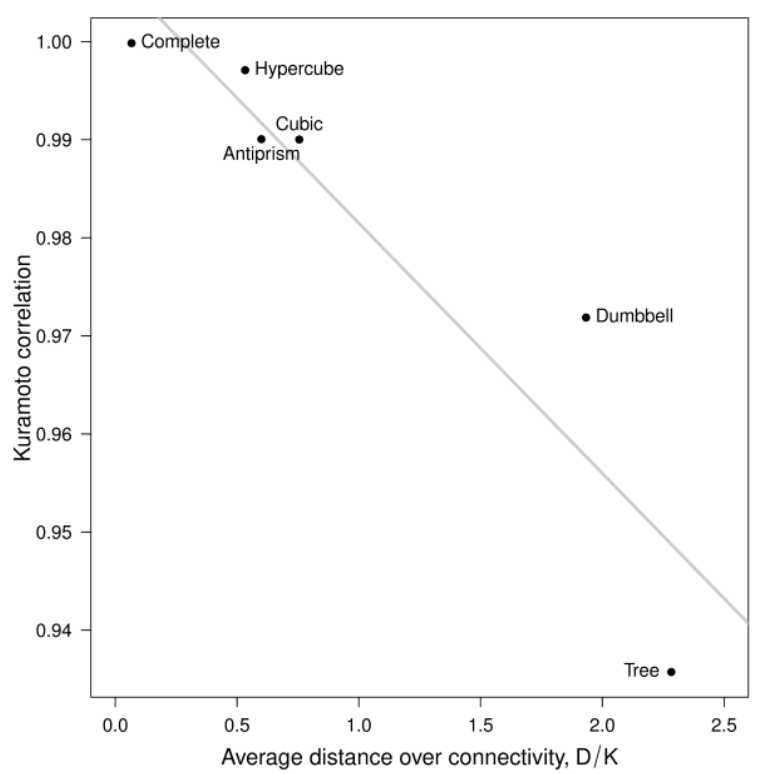

(b)

Figure 5. (a) Kuramoto Model dynamics calculated using the ode facility of the R toolkit, for the networks in Figure 2, with $k=0.1$, and for random $\omega_{i}$ and initial $\theta_{i}$, selecting the median final $r$ value out of 11 runs.

(b) Final $r$ value from (a) as a function of average distance $(D)$ divided by connectivity $(K)$.

What is the main lesson for $\mathrm{C} 2$ from the Kuramoto Model? As Figure 5 illustrates, it is that successful synchronisation depends on short network paths (i.e. a low average distance $D$ ) and multiple alternate network paths (i.e. a high connectivity $K$ ). Dekker (2010) explores this dependence in more detail. This lesson is not a particularly surprising one, since $D$ correlates with speed of transmission through a network, and $K$ is a measure of network robustness. However, it is interesting that the effect of network topology can largely be reduced to these two network metrics - other network properties have comparatively minor effects, although these may still be important (Dekker 2013b).

It should be noted that this is not the only possible application of the Kuramoto Model to C2. Kalloniatis (2008) interprets the phase angles $\theta_{i}$ as positions within an Observe-Orient-Decide-Act (OODA) loop. The correspondences in Table 1 and the empirical data discussed in this paper do not apply to that quite different construct, which provides a theory of synchronising friendly OODA loops and disrupting hostile ones.

\section{EXPERIMENT - METHOD}

The experiment reported here involved a simple combat simulation written in NetLogo (Wilensky 1999). Figure 6 shows a screenshot of the simulation. The blue force here consists of 8 infantry fighting vehicles (IFVs) and 8 artillery units, connected using one of the network topologies in Figure 2 (with nodes 1-8 being artillery and nodes 9-16 IFVs). The simulated network allows messages to be sent along communication links. The red force consists of 40 stationary units, each with the same combat power as the IFVs. The blue mission is to reach the east side of the battlefield in Figure 6.

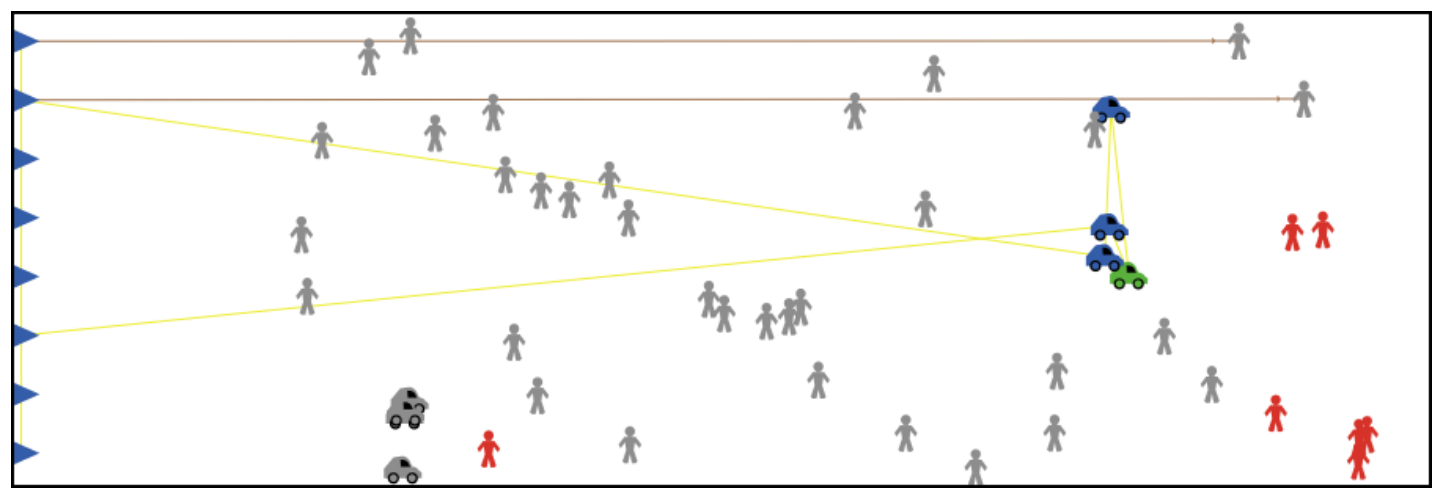

Figure 6. Screenshot of the NetLogo simulation. Triangles represent blue artillery, with brown arrows showing recent artillery strikes. Vehicles show blue infantry fighting vehicles (IFVs) advancing to the right, with the current leader highlighted in green. The blue communication network is shown in yellow. Human figures represent red units. Vehicles and human figures coloured grey have been killed. 
To achieve their mission, the blue IFVs elect a leader using a distributed consensus algorithm (Mullender, 1993). Specifically, each blue IFV broadcasts a message volunteering for the role, and the IFV with the lowest id number is selected as leader. The other blue IFVs then adjust their speed to remain west of the leader. Meanwhile, blue artillery supports the advance by laying down a barrage along a line of longitude just to the east of the leader. Clearly, communication failures can result in this barrage being too far to the east (thereby allowing red units to target the IFVs), or too far to the west (thereby also allowing blue-on-blue casualties).

For blue forces, the correlation (synchronisation) measure is the level of agreement on which vehicle is the leader. For a pair of units, this is 1 if they agree, and 0 if they do not. For the (surviving) force as a whole at a specific point in time, it is the average over all pairs of units. For a specific simulation run, it is the average for the force over all time steps. For a particular network, it is the average over 1,000 simulation runs.

The measure of mission success is the number of blue IFVs reaching the right (east) side of the battlefield in Figure 6. This is also averaged over 1,000 simulation runs for each network.

\section{EXPERIMENT - RESULTS}

Figure 7 shows that there is a monotonic nonlinear relationship between correlations in the Kuramoto Model (as per Figure 5) and plan-agreement correlations in the combat simulation for the corresponding network topology. This relationship is statistically significant $(p<1 \%)$. In other words, this confirms that synchronisation performance (correlation) of networks in the combat simulation corresponds to synchronisation performance (correlation) of networks in the Kuramoto Model.

The relationship between network topology and mission success is more complex. This is because, while synchronisation supports good mission performance, there are also direct effects of network topology on mission performance. Specifically, effective communication of position updates from the lead vehicle is necessary for accurate artillery support (a similar situation occurs in the real world, where the network is used both for synchronising plans and for targeting).

With regard to mission performance, the networks divide into two groups: the "poor" networks with connectivity $K=1$ (Tree and Dumbbell), for which a mean of 4.42 vehicles reach the goal; and the "good" networks with connectivity $K \geq 3$, for which a mean of 5.46 vehicles reach the goal (i.e. a mean of 1.04 additional vehicles). Overlap between confidence intervals does not permit distinguishing between networks within these two groups.

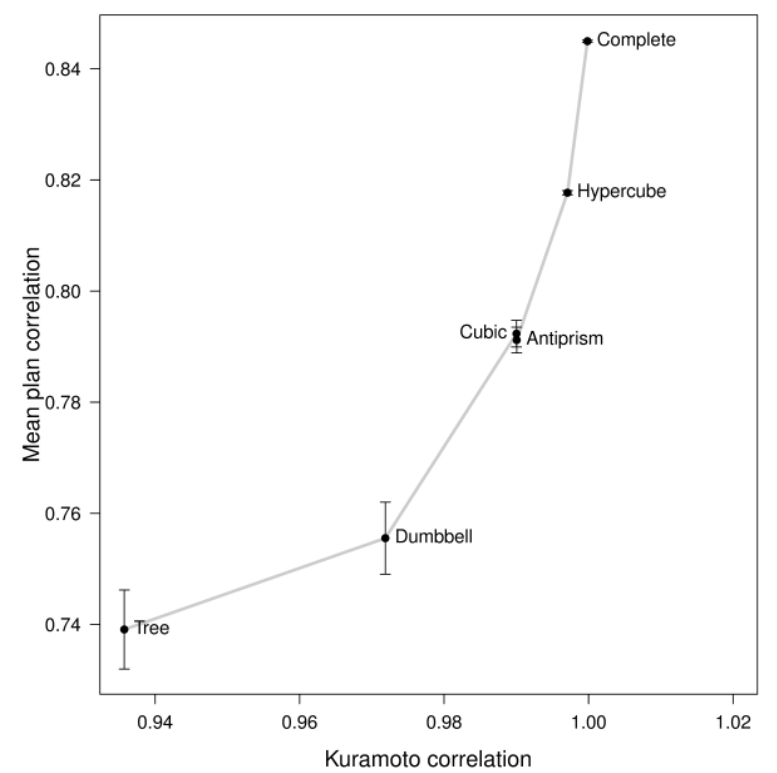

(a)

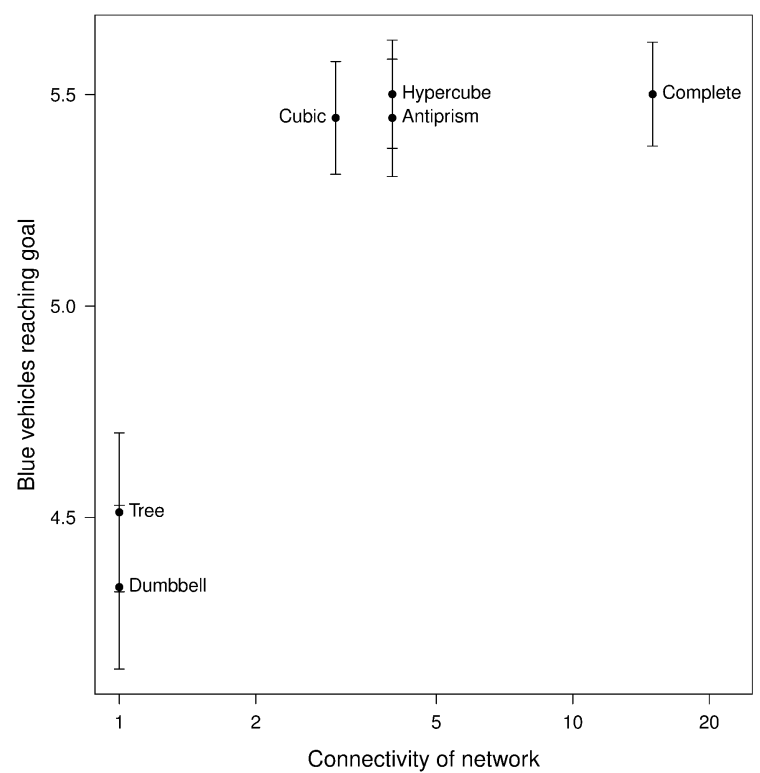

(b)

Figure 7. (a) Correlations in the combat simulation compared to correlations for the Kuramoto Model with the same network topology.

(b) Mission performance in the combat simulation compared to connectivity of the network topology. Error bars show $95 \%$ confidence intervals. 


\section{DISCUSSION}

The experimental results above underscore the relationship between Kuramoto Model dynamics and military synchronisation noted in Table 1, and hence the main lesson of the Kuramoto Model: that successful synchronisation depends on short network paths (i.e. a low average distance $D$ ) and on multiple alternate network paths (i.e. a high connectivity $K$ ). This lesson is not, of course, new. It has long been argued that short network paths speed up information-sharing, and that multiple alternate network paths improve robustness (see e.g. Alberts and Hayes 2003, 2006, 2007). Modern joint and coalition operations avoid parallel "stovepipe" C2 structures by supplementing tree topologies with additional links.

So what is the benefit in revisiting the Kuramoto Model in a C2 context? Its very simplicity makes it an illustration of how theoretical constructs should be used in models of human behaviour. As Agar (2003) points out, "The most important thing to translate when going from research to model are the human concepts that make the biggest difference in explaining what happens in the population. Then make sure the computer versions of the concepts interact like the human concepts did in the ethnographic explanation." This requires the existence of a mapping like the one in Table 1, and this mapping should be justified by experimental work, such as the experiment reported here.

There have been cases where theoretical constructs have been applied without such justification. For example, Maslow's "Hierarchy of Needs" (Maslow 1943, 1962) has been used in social simulation models of human behaviour in Iraq and Afghanistan. However, this hierarchy is subject to several criticisms. In particular, Dunne (2009) suggests that it is a very poor model of non-Western cultures, and is consequently "non-deployable" to countries like Iraq.

To be useful, a model must generate some kind of knowledge about the entity or process being modelled. Taking the classical definitions of knowledge as justified true belief and truth as corresponding to reality (Audi 2003; Dekker 2013a), this requires a justification both for the applicability of the model and for the relationship between model dynamics and real-world dynamics. Table 1 provides an example of such relationships - but the mappings in such a table must be based on evidence and testing, rather than simply on assertion.

Models may deviate from reality in two ways, both of which can be found in Table 1. First, there will be many aspects of reality that are not represented (or not adequately represented) in the model. No conclusions about those aspects of reality can therefore be drawn from the model. In the case of the Kuramoto Model, most aspects of synchronisation in military systems are unrepresented, and the model thus tells us nothing about them. From the point of view of a specific application, however, we would expect the model to represent the "most prominent causal relations" (Galán et al. 2009).

The second way in which a model may deviate from reality is by containing artefacts - properties of the model which do not correspond to properties of the real world. In the case of the Kuramoto Model, the difficulty in synchronising large cycles is such an artefact. Experiments with the Kuramoto Model do not provide any grounds for recommendations against network topologies containing large cycles. Developers of simulation models must be on guard against such artefacts. Confirming a valid correspondence between model and reality can be done using experiments in the field, or by using other simulation models of a different kind. In this paper, for example, we have shown how a simple agent-based simulation can be used to confirm the predictions of the Kuramoto Model.

There are other approaches to truth in simulation than the correspondence theory of truth that we have used (Schmid 2005). A consensus theory of truth takes a simulation as valid if a majority of stakeholders and subject-matter experts believe that it is. A coherence theory of truth takes a simulation as valid if it is consistent with other beliefs. Both of these approaches ignore "ground truth" and can therefore lead to "groupthink" and to an uncritical acceptance of simulation results. In particular, the deficiencies of Maslow's "Hierarchy of Needs" noted by Dunne (2009) would not have been recognised under those two approaches to truth. It is therefore preferable to rely on the correspondence theory of truth which underlies Table 1. Particularly with military simulations, where lives are on the line, it is important that decision-makers have confidence that their models do indeed reflect reality.

\section{REFERENCES}

Agar, M. (2003). My Kingdom for a Function: Modeling Misadventures of the Innumerate. Journal of Artificial Societies and Social Simulation, 6(3), 8: jasss.soc.surrey.ac.uk/6/3/8.html

Alberts, D.S. and Hayes, R.E. (2003). Power to the Edge, CCRP Publication Series, Washington, D.C., www.dodccrp.org/files/Alberts_Power.pdf 
Dekker, C2 and the Kuramoto Model: An Epistemological Retrospective

Alberts, D.S. and Hayes, R.E. (2006). Understanding Command and Control, CCRP Publication Series, Washington, D.C., www.dodccrp.org/files/Alberts_UC2.pdf

Alberts, D.S. and Hayes, R.E. (2007). Planning: Complex Endeavors, CCRP Publication Series, Washington, D.C., www.dodccrp.org/files/Alberts_Planning.pdf

Audi, R. (2003). Epistemology: A contemporary introduction to the theory of knowledge (2nd ed.). London, UK: Routledge.

Becker, J., Niehaves, B., \& Klose, K. (2005). A framework for epistemological perspectives on simulation. Journal of Artificial Societies and Social Simulation, 8(4), 1. jasss.soc.surrey.ac.uk/8/4/1.html

Cioffi-Revilla, C. (2010). A methodology for complex social simulations. Journal of Artificial Societies and Social Simulation, 13(1), 7: jasss.soc.surrey.ac.uk/13/1/7.html

Crawley, M. J. (2007). Dekker, A.H. (2007). Studying Organisational Topology with Simple Computational Models. Journal of Artificial Societies and Social Simulation, 10(4), 6: jasss.soc.surrey.ac.uk/10/4/6.html

Dekker, A.H. (2010). Average Distance as a Predictor of Synchronisability in Networks of Coupled Oscillators. ACSC2010, 33 ${ }^{\text {rd }}$ Australasian Computer Science Conference (CRPIT, Vol. 102), 127-131.

Dekker, A.H. (2013a). Epistemological Aspects of Simulation Models for Decision Support. International Journal of Agent Technologies and Systems, 5(2), 55-77. doi:10.4018/jats.2013040103

Dekker, A.H. (2013b). Self-Synchronisation in C2 Networks. In Piantadosi, J., Anderssen, R.S. and Boland J. (eds) MODSIM2013, $20^{\text {th }}$ International Congress on Modelling and Simulation, MSSANZ, 998-1004. www.mssanz.org.au/modsim2013/D1/dekker.pdf

Dorogovtsev, S.N., Goltsev, A.V., \& Mendes, J.F.F. (2008). Critical phenomena in complex networks. Reviews of Modern Physics, 80(4), 1275-1353. doi:10.1103/RevModPhys.80.1275

Dunne, J.P. (2009). Maslow is non-deployable: Modifying Maslow's hierarchy for contemporary counterinsurgency. In P. Holmes-Eber, P. M. Scanlon, \& A. L. Hamlen (Eds.), Applications in operational culture: Perspectives from the field, 15-28. Quantico, VA: Marine Corps University Press.

Galán, J. M., Izquierdo, L. R., Izquierdo, S. S., Santos, J. I., del Olmo, R., López-Paredes, A., \& Edmonds, B. (2009). Errors and Artefacts in Agent-Based Modelling. Journal of Artificial Societies and Social Simulation, 12(1), 1. jasss.soc.surrey.ac.uk/12/1/1.html

Grüne-Yanoff, T. and Weirich, P. (2010). The Philosophy and Epistemology of Simulation: A Review. Simulation \& Gaming, 41(1), 20-50. doi:10.1177/1046878109353470

Kalloniatis, A. (2008, June 17-19). A new paradigm for dynamical modelling of networked C2 processes. Proceedings of the $13^{\text {th }}$ International Command and Control Research and Technology Symposium, Seattle, WA. www.dodccrp.org/events/13th_iccrts_2008/CD/html/papers/180.pdf

Kalloniatis, A. (2010). From incoherence to synchronicity in the network Kuramoto Model. Physical Review E: Statistical, Nonlinear, and Soft Matter Physics, 82, 066202. doi:10.1103/Phys-RevE.82.066202

Kalloniatis, A. \& Zuparic, M. (2014), The J-Staff System, Network Synchronisation and Noise. Proceedings of the $19^{\text {th }}$ International Command and Control Research and Technology Symposium, Alexandria, VA.

Kalloniatis, A. \& Zuparic, M. (2016), Fixed points and stability in the two-network frustrated Kuramoto model. Physica A, 447, 21-35, doi:10.1016/j.physa.2015.11.021

Kuramoto, Y. (1984). Chemical Oscillations, Waves, and Turbulence, Berlin: Springer.

Maslow, A.H. (1943). A theory of human motivation. Psychological Review, 50(4), 370-396. doi:10.1037/h0054346

Maslow, A.H. (1962). Toward a psychology of being. New York: D. Van Nostrand. doi:10.1037/10793-000

Mullender, S.J. (1993). Distributed Systems. ACM Press.

Schmid, A. (2005). What is the truth of simulation? Journal of Artificial Societies and Social Simulation, 8(4), 5: jasss.soc.surrey.ac.uk/8/4/5.html.

Strogatz, S.H. (2000). From Kuramoto to Crawford: exploring the onset of synchronization in populations of coupled oscillators. Physica D. Nonlinear Phenomena, 143, 1-20. doi:10.1016/S0167-2789(00)00094-4

Wilensky, U. (1999). NetLogo. Center for Connected Learning and Computer-Based Modeling, Northwestern University, Evanston, IL. ccl.northwestern.edu/netlogo

Winfree, A.T. (1980). The Geometry of Biological Time, New York: Springer. 